\title{
Clinical Assessment of MR-Guided 3-Class and 4-Class Attenuation Correction in PET/MR
}

\author{
Hossein Arabi, ${ }^{1}$ Olivier Rager, ${ }^{1}$ Asma Alem, ${ }^{1}$ Arthur Varoquaux, ${ }^{2}$ Minerva Becker, ${ }^{2}$ \\ Habib Zaidi ${ }^{1,3,4}$ \\ ${ }^{1}$ Division of Nuclear Medicine and Molecular Imaging, Department of Medical Imaging, Geneva University Hospital, CH-1211, Geneva, \\ Switzerland \\ ${ }^{2}$ Division of Diagnostic Radiology, Department of Medical Imaging, Geneva University Hospital, CH-1211, Geneva, Switzerland \\ ${ }^{3}$ Geneva Neuroscience Center, Geneva University, CH-1205, Geneva, Switzerland \\ ${ }^{4}$ Department of Nuclear Medicine and Molecular Imaging, University of Groningen, University Medical Center Groningen, 9700 RB, \\ Groningen, Netherlands
}

\begin{abstract}
Purpose: We compare the quantitative accuracy of magnetic resonance imaging (MRI)-based attenuation correction $(A C)$ using the 3-class attenuation map (PET-MRAC3c) implemented on the Ingenuity TF PET/MRI and the 4-class attenuation map (PET-MRAC4C) similar to the approach used on the Siemens mMR PET/MR considering CT-based attenuation-corrected PET images (PET-CTAC) as standard of reference.

Procedures: Fourteen patients with malignant tumors underwent whole-body sequential 2deoxy-2-[ $\left.{ }^{18} \mathrm{~F}\right]$ fluoro-D-glucose $\left({ }^{18} \mathrm{~F}-\mathrm{FDG}\right)$ positron emission tomography (PET)/X-ray computed tomography (CT) and PET/MR imaging. A 3-class attenuation map was obtained from segmentation of T1-weighted MR images followed by assignment of attenuation coefficients (air $0 \mathrm{~cm}^{-1}$, lung $0.022 \mathrm{~cm}^{-1}$, soft tissue $0.096 \mathrm{~cm}^{-1}$ ), whereas a 4-class attenuation map was derived from a MR Dixon sequence (air $0 \mathrm{~cm}^{-1}$, lung $0.018 \mathrm{~cm}^{-1}$, fat $0.086 \mathrm{~cm}^{-1}$, soft tissue $0.096 \mathrm{~cm}^{-1}$ ). Additional adipose tissue class and inner body air cavities (e.g., sinus and abdomen) were also considered. Different attenuation coefficients were assigned to the lungs since the two techniques were implemented as they were proposed without any modification.

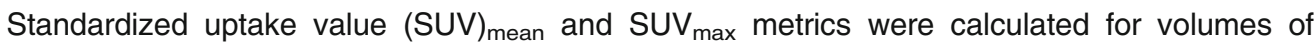
interest in various organs/tissues and malignant lesions. Well-established metrics were used for the analysis of SUVs estimated using both PET-MRAC techniques and PET-CTAC including relative error, Spearman rank correlation, and Bland and Altman analysis.

Results: PET-MRAC3C and PET-MRAC4c revealed significant underestimation of SUV for normal organs $(-17.4 \pm 8.5$ and $-22.0 \pm 6.8 \%$, respectively) compared to PET-CTAC. Lesions' SUV presented the same trend with larger underestimation for PET-MRAC4C $(-9.2 \pm 6.1 \%)$ compared to PET-MRAC3c $(-3.9 \pm 9.0)$. The different attenuation coefficients assigned to the lungs with both techniques resulted in significant positive bias on PET-MRAC3c (18.6 $\pm 15.3 \%)$ and low negative bias on PET-MRAC4c $(-0.5 \pm 13.3 \%)$. Both approaches yielded the largest differences in and near bony structures. Despite the large bias, there was good correlation between PET-MRAC3c $(R=0.97$, $P<0.01)$ and PET-CTAC, and PET-MRAC4c $(R=0.97, P<0.01)$ and PET-CTAC, respectively.
\end{abstract}

Electronic supplementary material The online version of this article (doi:10.1007/s11307-014-0777-5) contains supplementary material, which is available to authorized users.

Correspondence to: Habib Zaidi; e-mail: habib.zaidi@hcuge.ch 
Conclusions: PET-MRAC3c resulted in significant systematic positive bias in the lungs owing to the lower attenuation coefficient used and negative bias in other regions. PET-MRAC4c slightly underestimated tracer uptake in the lungs and led to even larger negative bias than PETMRAC3c in other body regions. The presence of artifacts in the MRAC might lead to misinterpretation of clinical studies. As such, the attenuation map needs to be checked for artifacts as part of the reading procedure to avoid misinterpretation of SUV measurements.

Key words: PET/MRI, PET/CT, Attenuation correction, SUV, Quantification

\section{Introduction}

$\mathrm{T}^{\mathrm{s}}$ he growing clinical and research interest in hybrid positron emission tomography (PET)/magnetic resonance (MR) imaging demonstrate that this technology could provide a major technological breakthrough having the potential to trigger a ground-breaking paradigm shift in clinical diagnostic imaging and revolutionize clinical practice [1]. There are many compelling reasons to combine PET and MRI such as higher soft tissue contrast compared with X-ray computed tomography (CT), the availability of various MRI sequences, and the foremost advantage of MRI is the absence of radiation exposure [2, 3]. Therefore, much research efforts are directed toward the development of combined PET/MR systems and addressing related crucial issues such as quantitative imaging [4].

Although the preliminary clinical results obtained using combined PET/MR are promising [5-7], attenuation correction is technically challenging since the MR signal is not correlated with attenuation coefficients of biological tissues. Nevertheless, a number of approaches have been suggested to generate an attenuation map from MR images [8, 9]. This includes MR image segmentation techniques [10-12], atlas and machine learning approaches $[13,14]$, the use of special MRI sequences such as ultra-short echo [15-17], and joint estimation of emission and transmission maps through iterative reconstruction of emission data only [18-20].

Segmentation-based methods, the most widely employed techniques, rely on classifying the patient MR image into a number of tissue classes followed by assignment of predefined linear attenuation coefficients. However, ignoring bony structures is the major drawback of these techniques; since the percentage of bone tissue in the body is relatively low compared to specific body regions/organs such as the head, attenuation correction that neglects bones may be sufficient for most clinical indications [11, 12]. Yet, underestimation of tracer uptake by osseous lesions has been reported in a number of studies, typically ranging within 5-8 \% [11, 12, 21] or up to $15 \%$ [22] and $23 \%$ [23] compared to what is achieved using clinical PET/CT data, whereas simulation studies using an anthropomorphic thorax phantom reported a local bias of up to $17 \%$ [24] and even $30 \%$ [25]. The average underestimation of tracer uptake in bony structure was reported to vary between $7.8 \%$ [11] and $27 \%$ [14]. Comparison of segmentation-based attenuation correction methods revealed underestimation of tracer uptake by $15-16 \%$ when using a 3-class attenuation map [26]. These discrepancies can be partially explained by the assignment of different attenuation coefficients for each individual class. For instance, a linear attenuation coefficient of $0.101 \mathrm{~cm}^{-1}$ for soft tissue was used in one study [14], whereas $0.0952 \mathrm{~cm}^{-1}$ was used in another $[22,26]$.

The Philips Ingenuity TF PET/MR (Philips Healthcare, Cleveland, OH, USA) uses a 3-class attenuation map from segmentation of a T1-weighted MR sequence including air, lung, and soft tissue $[12,27]$. Conversely, the approach used on the Siemens mMR PET/MR (Siemens Healthcare, Erlangen, USA) system generates a 4-class attenuation map from segmentation of a Dixon MR sequence [28] including fat in addition to the above-referenced tissue classes [11, 29]. The above-referenced MRAC procedures are widely used in clinical setting. Therefore, the clinical assessment of their performance is commended.

In this work, we compare the 3-class (air, lung, soft tissue) and the 4-class (air, lung, fat, soft tissue) approaches implemented on the Ingenuity TF PET/MRI and reported by Martinez-Moller et al. [11] (an approach similar to the technique implemented on the Biograph mMR PET/MR), respectively, using clinical whole-body PET data. PET images corrected for attenuation using co-registered CT images are used as reference for comparison of the standardized uptake values (SUVs).

\section{Materials and Methods}

\section{PET/CT and PET/MR Data Acquisition}

The study population comprised 14 consecutive patients, 9 men and 5 women (mean age $\pm \mathrm{SD}=61 \pm 7$ years), in whom MRI of the head and neck, whole-body 2-deoxy-2-[ ${ }^{18}$ F $]$ fluoro-D-glucose $\left({ }^{18} \mathrm{~F}-\mathrm{FDG}\right)$ $\mathrm{PET} / \mathrm{MR}$, and whole-body ${ }^{18} \mathrm{~F}$-FDG PET/CT were performed for staging of head and neck malignancies. The study protocol was approved by the institutional ethics committee and all patients gave informed consent to participate in the study. A single injection of ${ }^{18}$ F-FDG $(371 \pm 23 \mathrm{MBq})$ was used.

${ }^{18}$ F-FDG PET/CT scans were performed on a Biograph 64 True Point scanner (Siemens Healthcare, Erlangen, Germany) After a 
localization scout scan, an unenhanced CT scan (120 kVp, $180 \mathrm{mAs}, 24 \times 1.5$ collimation, a pitch of 1.2 , and $1 \mathrm{~s}$ per rotation) was performed for attenuation correction. PET data acquisition started $146.2 \pm 20 \mathrm{~min}$ post-injection with $3 \mathrm{~min}$ per bed position for a total of 5-6 beds, resulting in a total acquisition time of 15-18 $\mathrm{min}$.

MRI examinations were performed on the Ingenuity TF PET/ MR [27] The so-called atMR whole-body MRI sequence was developed for fast acquisition $(<3 \mathrm{~min})$ of the 3 -class attenuation map. It consists of a 3D multi-stack spoiled T1-weighted gradient echo sequence with the following parameters: flip angle $10^{\circ}, \mathrm{TE}$ $2.3 \mathrm{~ms}$, TR $4.1 \mathrm{~ms}$, smallest water-fat shift, 600-mm transverse FOV with a slab thickness of $120 \mathrm{~mm}$, voxel size $1.9 \times 1.9 \times 6 \mathrm{~mm}^{3}$, and 12-mm overlap between adjacent stacks [12]. The 4-class attenuation map (air, lung, fat, and soft tissue) is derived from a Dixon sequence $[11,30]$. The whole-body Dixon 3D volumetric interpolated $\mathrm{T} 1$-weighted sequence used the following parameters: flip angle $10^{\circ}, \mathrm{TE}_{1} 1.1 \mathrm{~ms}, \mathrm{TE}_{2} 2.0 \mathrm{~ms}$, TR $3.2 \mathrm{~ms}, 450 \times 354 \mathrm{~mm}^{2}$ transverse FOV, $0.85 \times 0.85 \times 3 \mathrm{~mm}^{3}$ voxel size, and a total acquisition time of $2 \mathrm{~min}$ and $17 \mathrm{~s}$. It should be noted that the Dixon sequence parameters used in this work are slightly different from what has been used in $[9,29]$ ( $\mathrm{TE}_{1} 1.17 \mathrm{~ms}$ and TR $20 \mathrm{~ms}$ ), which might result in slightly different fat and water contrast, thus impacting the segmentation results. In-phase images were used for anatomic localization and PET/MR data fusion and registration. The maximum temporal difference between PET/CT and PET/MR scans was less than an hour.

\section{Attenuation Correction}

Dixon MR images (in-phase) were deformably registered to the corresponding CT images obtained from the PET/CT scan using the Elastix package (based on the ITK library) [31]. The alignment was performed by employing a combination of rigid and non-rigid registration based on maximum mutual and B-spline transform as described previously [32]. A multi-resolution approach with six steps from $1 / 32$ to $1 / 1$ along with a stochastic gradient descent optimizer was utilized for co-registration of in-phase MR to CT. Thirty-two histogram bins and 2,000 iterations at each resolution achieved good results. The obtained transformation fields were applied to atMR images taking advantage of prior alignment of Dixon and atMR images. It should be noted that the regions located in the vicinity of misalignment errors were disregarded from further analysis.

Subsequently, the co-registered atMR images were used to create a 3-class attenuation map corresponding to air (background), lung, and soft tissue according to the method implemented on the Ingenuity PET/MR system with the following attenuation coefficients: $0 \mathrm{~cm}^{-1}(-1,000 \mathrm{HU})$, $0.022 \mathrm{~cm}^{-1}(-770 \mathrm{HU})$, and $0.096 \mathrm{~cm}^{-1}(0 \mathrm{HU})$ for background, lung, and soft tissue, respectively [33].

Likewise, a 4-class segmentation of MR images (air, lung, fat, and soft tissue) was carried out using in-phase and fat-only Dixon images. In the first step, the transformation matrices obtained from the registration of in-phase images to the corresponding CT images were applied to register the corresponding fat-only MR images. The external body contour and lungs were determined by applying a 3D snake active contour algorithm [34]. The segmentation was performed through connected-component analysis of the low intensity voxels initiated by manual seeds using the ITK-SNAP image processing software [35]. In the next step, fat masks obtained using histogram-based thresholding of fat-only images [36] were superimposed on the segmented in-phase images to achieve a 4class segmented image to which the following attenuation coefficients were assigned: air $0 \mathrm{~cm}^{-1}(-1,000 \mathrm{HU})$, lung $0.018 \mathrm{~cm}^{-1}(-820 \mathrm{HU})$, fat $0.086 \mathrm{~cm}^{-1}(-140 \mathrm{HU})$, and soft tissue $0.096 \mathrm{~cm}^{-1}(0 \mathrm{HU})$. The procedure followed to derive the 4-class attenuation map was similar to the one proposed by MartinezMoller et al. [11] deemed to be the basis of the technique implemented on the Siemens mMR, although the latter approach seems to be specifically optimized for this scanner configuration using 4-class (with different attenuation coefficients) [29] or 5-class [9] attenuation maps. In the last step, PET/CT patient bed was then added to the 3- and 4-class attenuation maps followed by 3D Gaussian filtering (FWHM of $5 \mathrm{~mm}$ ). It is worth highlighting that the air gaps in the body (air-filled paranasal sinuses and gastrointestinal air) were also separately identified and assigned the corresponding attenuation coefficient. Segmentation of air sinus and air gaps in the abdomen was performed using an active contour technique initialized by manual selection of initial seeds in the air cavities.

\section{Image Reconstruction}

Image reconstruction was performed three times for each clinical study: PET images of the PET/CT data corrected for attenuation using CT (PET-CTAC) used as reference, PET attenuation corrected using the 3-class (PET-MRAC3c), and 4class (PET-MRAC4c) attenuation maps. PET image reconstruction was performed using attenuation-weighted, ordered subsetexpectation maximization (AW-OSEM) iterative reconstruction algorithm using default parameters (4 iterations, 8 subsets, and a post-processing Gaussian kernel with a FWHM of $5 \mathrm{~mm}$ ) on the multimodality workstation (Siemens Healthcare, Hoffman Estates, IL, USA).

\section{Data Analysis}

The comparison of PET images corrected for attenuation using the three different techniques was performed using volume of interest (VOI)-based analysis. A nuclear medicine physician drew manually the VOIs on regions of normal physiologic uptake and on areas of abnormally increased activity accumulation (lesions). The VOIs were defined on six regions in the lungs, liver, spleen, cerebellum, two bone tissues (cervical vertebrae 6 and dorsal vertebrae 5), aorta (as normal physiologic uptake), and malignant lesions. The differences between the attenuation correction techniques were quantified in terms of changes in the standard uptake value (SUV). The SUVs were calculated by dividing the activity concentration in each VOI by the injected activity divided by body weight (Eq. 1) [37]. For all defined VOIs, mean and max SUVs were measured to assess the strength of correlation between SUVs derived from PETCTAC and PET-MRAC images separately for different organs and malignant lesions. 


$$
\begin{aligned}
\mathrm{SUV}_{\text {mean }(\max )}= & \frac{\text { Mean }(\mathrm{Max}) \operatorname{VOI} \text { activity }(\mathrm{MBq} / \mathrm{ml})}{\operatorname{Injected~dose}(\mathrm{MBq})} \\
& \times \frac{\text { Body weight }(\mathrm{g})}{\text { decay factor of } \mathrm{F}^{18}}
\end{aligned}
$$

Of the 168 VOIs defined in normal physiologic regions, 146 VOIs were used for further evaluation, whereas 22 VOIs were excluded owing to prevalent errors, such as misalignment, metal artifacts, patient movement, and segmentation errors. In addition, of the 35 VOIs defined on malignant lesions, 4 were excluded for subsequent statistical analysis due to the same aforementioned reasons. The number of malignant lesions varied only slightly between the patients (range 2-4 lesions, mean=3).
Relative errors between SUVs measured on PET-MRAC3c/ PET-MRAC4c and PET-CTAC, used as reference, were computed for $\mathrm{SUV}_{\text {mean(max) }}$ according to Eq. (2). Subsequently, the average and standard deviation (SD) of relative errors were calculated for each individual VOI over all patients. In addition, the relative error was calculated on a voxel-by-voxel basis to ease the comparative evaluation process.

Relative error $(\%)$

$$
=\frac{\operatorname{MRAC}(\mathrm{SUV})-\text { Reference }(\mathrm{SUV})}{\text { Reference }(\mathrm{SUV})} \times 100 \%
$$

The Shapiro-Wilk test was used to examine null hypothesis that measured SUVs follow a normally distributed population and the
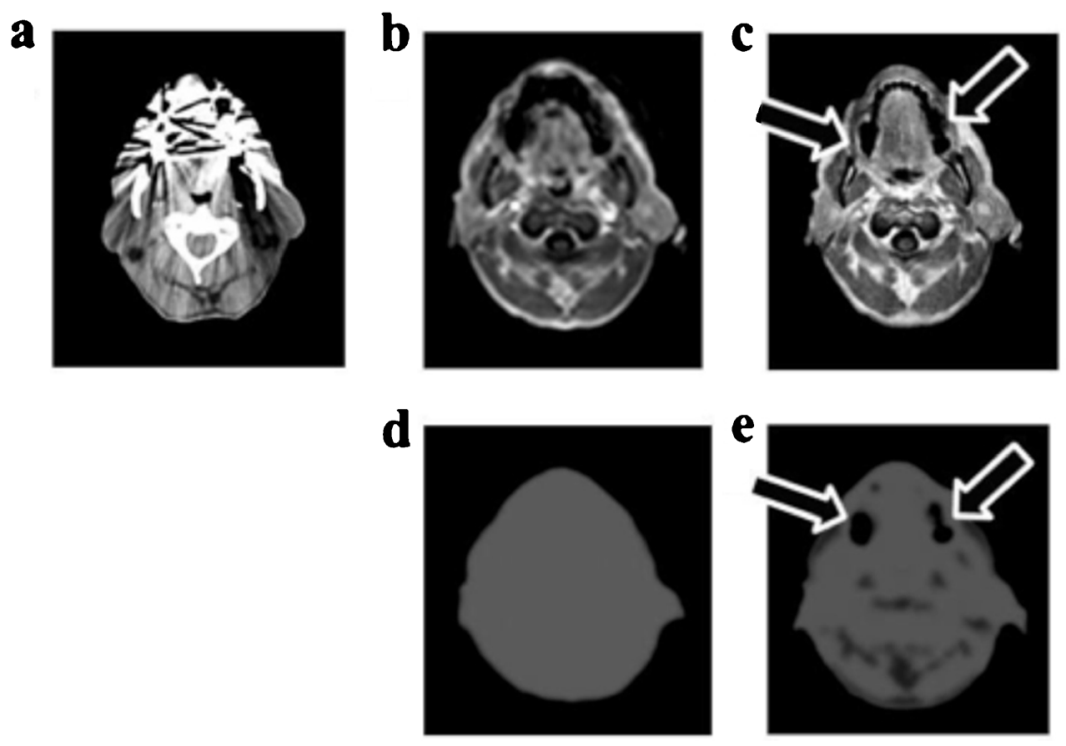

f

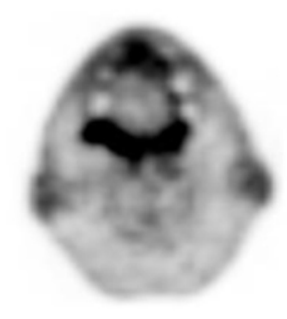

g
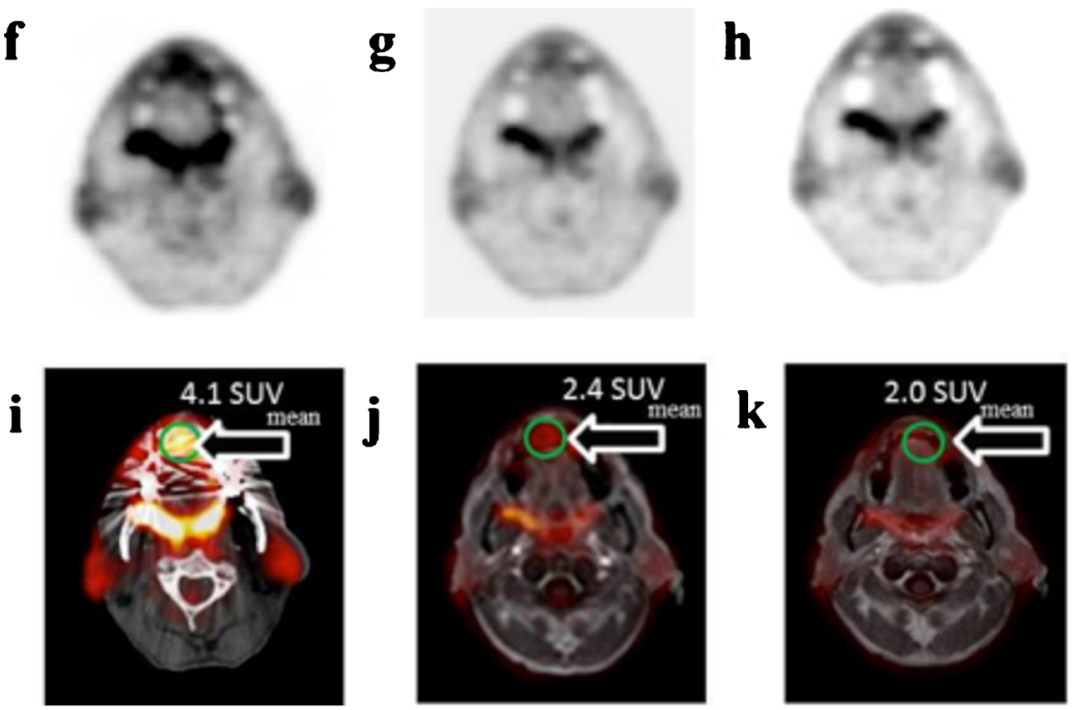

Fig. 1. Representative artifacts on PET-CTAC and PET-MRAC images due to the presence of metallic dental implants and their impact on SUV measurements in a patient with a squamous cell carcinoma of the retromolar trigone but no lesion in the floor of the mouth. a CT, $\mathbf{b}$ atMR, $\mathbf{c}$ Dixon, d MRAC3c, e MRAC4c, $\mathbf{f}$ PET-CTAC, $\mathbf{g}$ PET-MRAC3c, and $\mathbf{h}$ PET-MRAC4c, i fusion of PET-CTAC and CT, $\mathbf{j}$ fusion of PET-MRAC3c and atMR, and $\mathbf{k}$ fusion of PET-MRAC4c and Dixon. Note that a lesion with high metabolism appears to be present in the floor of the mouth on PET-CT, whereas no lesion is seen on PET/MR. 
Table 1. Relative errors between $\mathrm{SUV}_{\text {mean }}$ estimated using PET images corrected for attenuation using 3- (MRAC3c) and 4-class (MRAC4c)-based MRguided attenuation correction compared to CT-based attenuation correction (CTAC). Shapiro-Wilk test $P$ value $<0.05$ are considered statistically significant

\begin{tabular}{|c|c|c|c|c|}
\hline Region & MRAC $3 \mathrm{c}_{\text {mean }}$ & $P$ value & MRAC $4 \mathrm{c}_{\text {mean }}$ & $P$ value \\
\hline Upper right lung & $23.3 \pm 10.6$ & $<0.01$ & $-00.4 \pm 12.4$ & 0.24 \\
\hline Upper left lung & $19.1 \pm 11.4$ & $<0.01$ & $00.7 \pm 11.6$ & 0.33 \\
\hline Middle right lung & $20.0 \pm 16.1$ & $<0.01$ & $01.7 \pm 14.0$ & 0.29 \\
\hline Middle left lung & $21.5 \pm 18.9$ & 0.02 & $06.3 \pm 15.8$ & 0.45 \\
\hline Lower right lung & $22.6 \pm 20.2$ & 0.02 & $-01.0 \pm 14.4$ & 0.21 \\
\hline Lower left lung & $04.6 \pm 14.5$ & 0.49 & $-10.0 \pm 11.5$ & 0.06 \\
\hline Cerebellum & $-13.4 \pm 06.0$ & $<0.01$ & $-19.6 \pm 05.8$ & $<0.01$ \\
\hline Aorta cross & $-16.6 \pm 08.2$ & $<0.01$ & $-20.4 \pm 07.9$ & $<0.01$ \\
\hline Liver & $-10.2 \pm 06.7$ & $<0.01$ & $-14.7 \pm 03.4$ & $<0.01$ \\
\hline Spleen & $-10.7 \pm 10.0$ & 0.01 & $-16.3 \pm 07.6$ & $<0.01$ \\
\hline Bone (cervical 6) & $-29.3 \pm 09.4$ & $<0.01$ & $-34.5 \pm 07.0$ & $<0.01$ \\
\hline Bone (dorsal 5) & $-24.1 \pm 10.4$ & $<0.01$ & $-26.7 \pm 09.3$ & $<0.01$ \\
\hline Lesions & $-03.9 \pm 09.0$ & 0.01 & $-09.2 \pm 06.1$ & $<0.01$ \\
\hline
\end{tabular}

calculated $P$ values were reported for each individual VOI. Furthermore, the Spearman rank correlation coefficient $(\rho)$ was calculated to assess the correlation between SUVs measured on both PET-MRAC images and PET-CTAC according to Eq. (3).
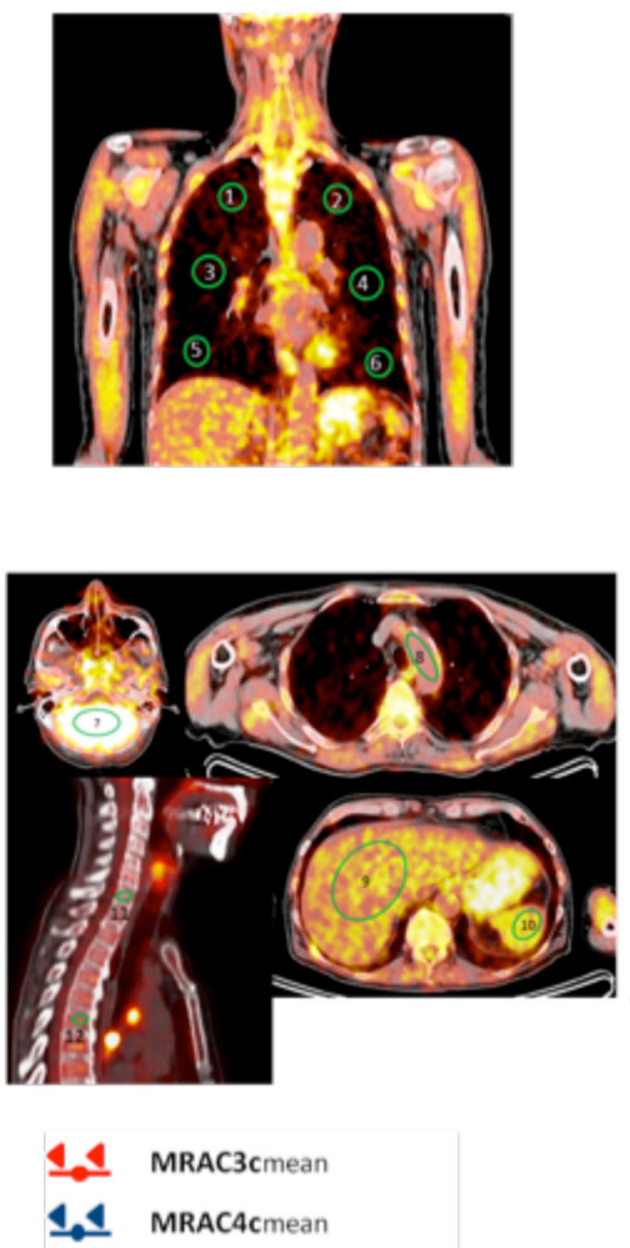

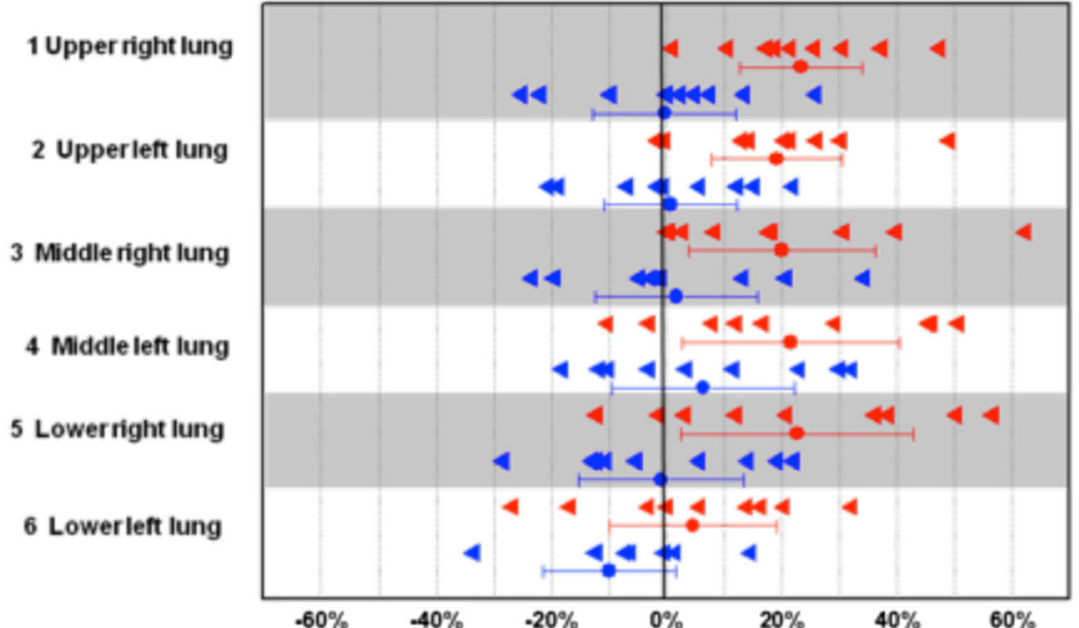

7 Cerebellum
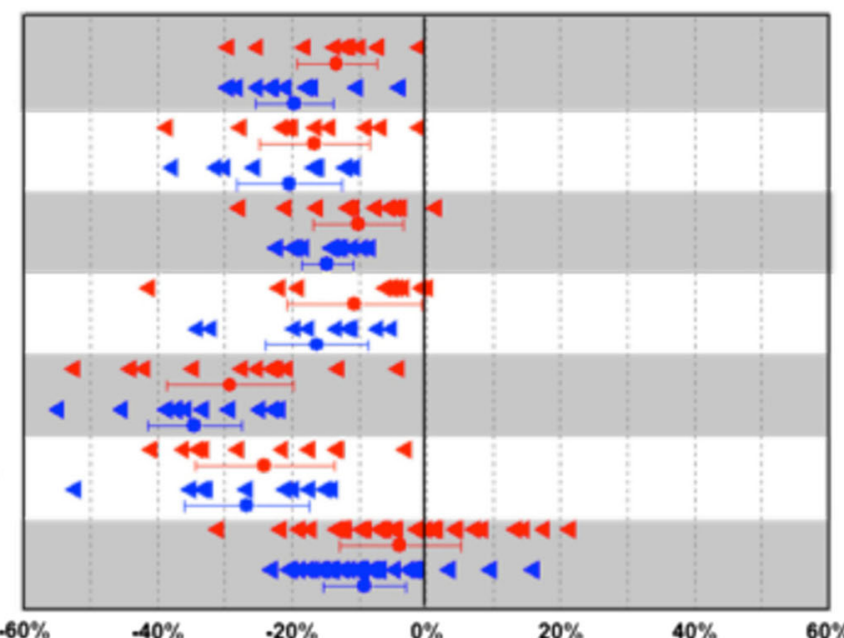

Lesion

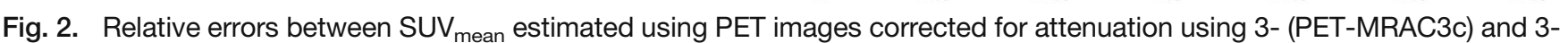
class (PET-MRAC4c)-based MR-guided attenuation correction compared to CT-based attenuation correction (PET-CTAC) for different organs. The dot point and line bar indicate the mean and standard deviation of the results, respectively. 
$\rho=\frac{\sum_{i}\left(M_{i}-\bar{M}\right)\left(C_{i}-\bar{C}\right)}{\sqrt{\sum_{i}\left(M_{i}-\bar{M}\right)^{2}\left(C_{i}-\bar{C}\right)^{2}}}$

Where $M$ and $C$ are measured SUVs on PET-MRAC and PETCTAC, respectively, and $i$ is the VOI number. Bland and Altman analysis [38] was used to determine the concordance between SUVs measured on both attenuation-corrected images by plotting PET-MRAC against PET-CTAC. Linear mixed models with a random effect on patients for non-normal distributed samples of clustered data were used to calculate the relative difference between SUVs from PET/MR and PET/CT in order to avoid bias from multiple lesions per patient $[39,40]$. The relative error might be high in regions with low tracer uptake, such as the lungs, whereas the absolute difference is quite insignificant. The absolute difference obtained by subtracting SUVs measured on PET-CTACs and PET-MRAC images were plotted for all organs and lesions.

\section{Results}

Visual inspection of PET/CT and PET/MR images of the 14 patients in terms of image quality, fusion accuracy, and lesions conspicuity was performed (Supplemental Fig. S1).

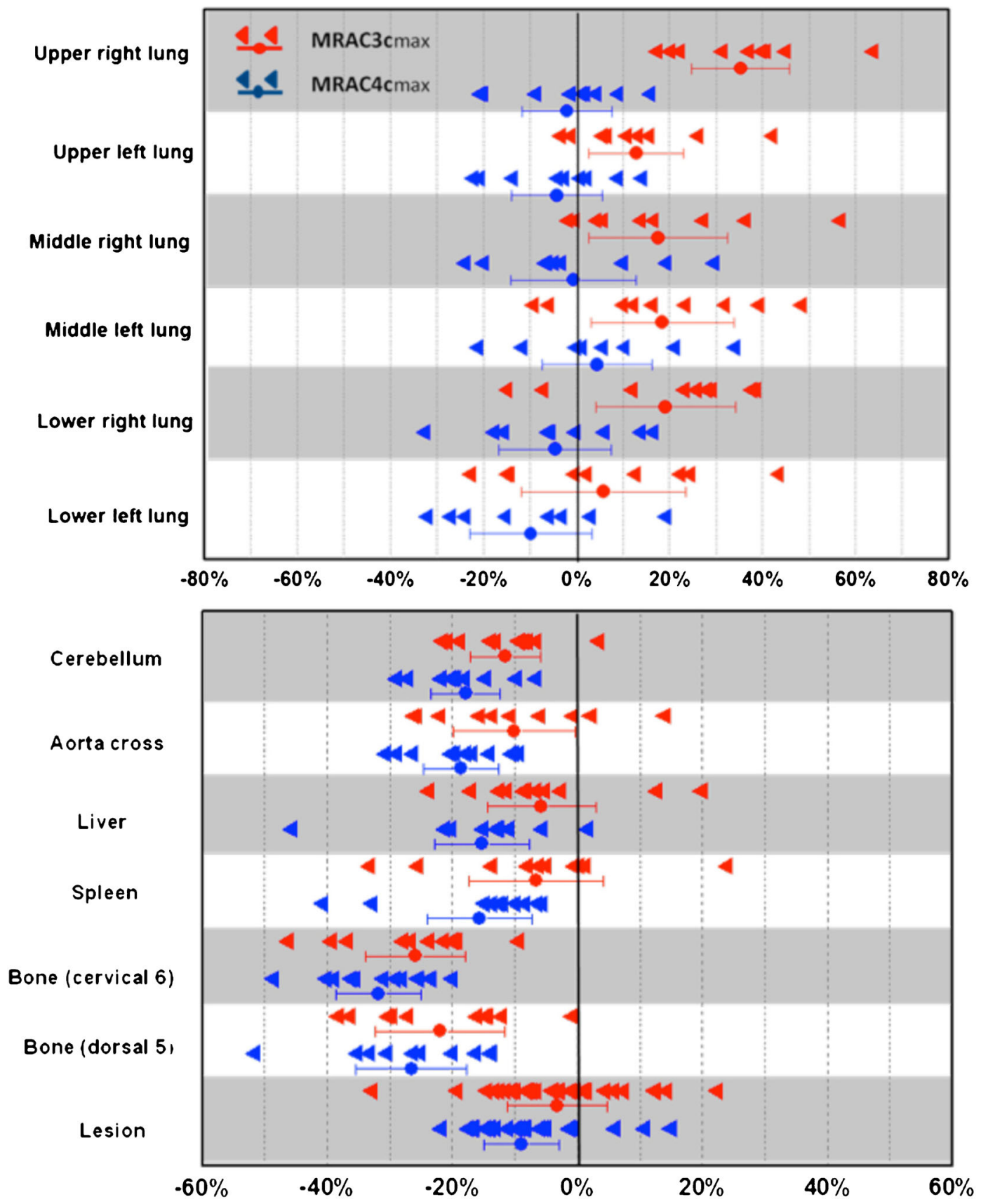

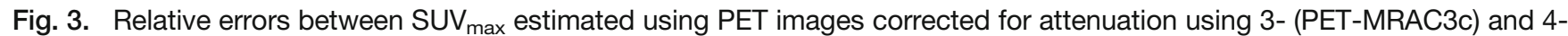
class (PET-MRAC4c)-based MR-guided attenuation correction compared to CT-based attenuation correction (PET-CTAC) for various organs. The dot point and line bar indicate the mean and standard deviation of the results, respectively. 
Table 2. Relative errors between $\mathrm{SUV}_{\max }$ estimated using PET images corrected for attenuation using 3- (MRAC3c) and 4-class (MRAC4c)-based MRguided attenuation correction compared to CT-based attenuation correction (CTAC). Shapiro-Wilk test $P$ values $<0.05$ are considered statistically significant

\begin{tabular}{llll}
\hline Region & MRAC3c $\mathrm{c}_{\max }$ & $P$ value & MRAC4c $_{\max }$ \\
\hline Upper right lung & $35.3 \pm 11.2$ & $<0.01$ & $-02.0 \pm 09.6$ \\
Upper left lung & $12.9 \pm 10.2$ & $<0.01$ & $-04.1 \pm 09.8$ \\
Middle right lung & $17.6 \pm 15.0$ & $<0.01$ & $-00.6 \pm 13.5$ \\
Middle left lung & $18.5 \pm 15.4$ & 0.02 & $0.5 \pm 11.8$ \\
Lower right lung & $19.2 \pm 15.0$ & 0.01 & $-04.6 \pm 12.1$ \\
Lower left lung & $05.9 \pm 17.7$ & 0.34 & $-09.8 \pm 13.1$ \\
Cerebellum & $-11.5 \pm 05.6$ & $<0.01$ & $-17.8 \pm 05.5$ \\
Aorta cross & $-10.1 \pm 09.8$ & 0.01 & $-18.6 \pm 06.0$ \\
Liver & $-05.8 \pm 08.7$ & 0.11 & $-15.3 \pm 07.5$ \\
Spleen & $-06.6 \pm 10.8$ & 0.1 & $-15.7 \pm 08.4$ \\
Bone (cervical 6) & $-25.8 \pm 07.9$ & $<0.01$ & $-31.8 \pm 06.7$ \\
Bone (dorsal 5) & $-22.0 \pm 10.4$ & $<0.01$ & $-26.6 \pm 8.8$ \\
Lesions & $-03.3 \pm 08.0$ & 0.01 & -0.13 \\
\end{tabular}

In the case of local mismatch or severe effect of deep breathing motion, the VOIs in the vicinity of the mismatch were excluded from further analysis. Three patients were affected by local mismatch in the cerebellum, lungs, and liver region and 1 patient presented with deep breathing motion. VOIs located in the vicinity of the misaligned regions were excluded from analysis. Dental fillings and implants degraded image quality on both PET-CTAC and
PET-MRAC images in 8 and 6 patients, respectively, and the ensuing metal artifacts impaired PET quantification, thus leading to considerable changes of tracer uptake (Fig. 1).

The procedure used to generate the 3-class attenuation map disregards air gaps and areas with no MR signal inside the body which generates an attenuation map where air gaps are filled with soft tissue (Fig. 1d). On the other hand, the procedure followed to generate the 4-class attenuation map
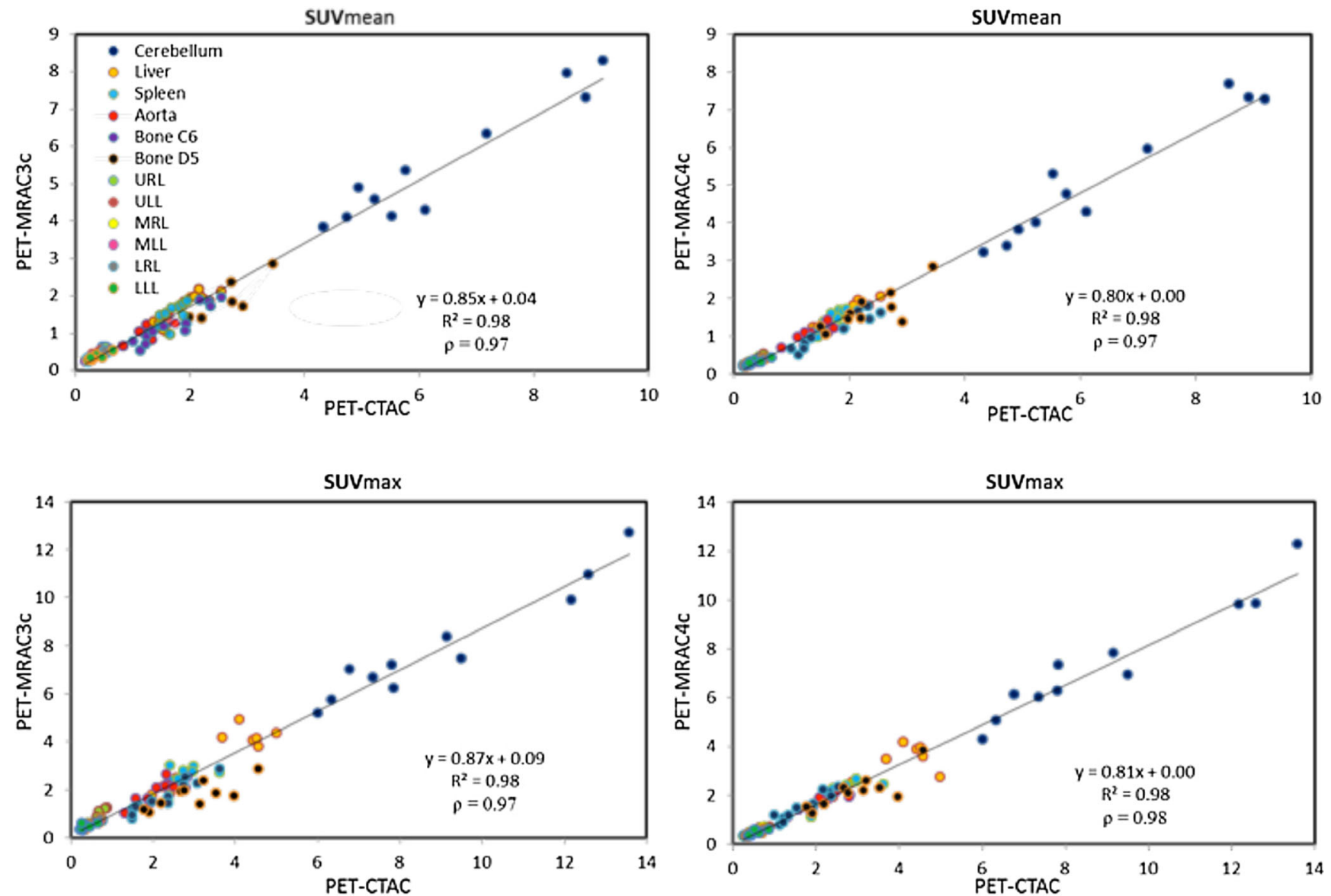

Fig. 4. Regression plots between $S U V_{\text {mean }} / S U V_{\text {max }}$ estimated using images corrected for attenuation using 3- (PET-MRAC3c) and 4-class (PET-MRAC4c)-based MR-guided attenuation correction and CT-based attenuation correction (PET-CTAC) for various organs. 
takes air gaps into account; however, MR image degradation due to the presence of metal implants may affect the 4-class attenuation map in most cases as shown in Fig. 1e. Table 1 summarizes the relative errors for $\mathrm{SUV}_{\text {mean }}$ between PETMRAC3c/PET-MRAC4c and PET-CTAC images (Fig. 2). The statistical significance ( $P$ value) of the Shapiro-Wilk test was also calculated for each VOI (statistical significance level at 0.05).

The relative $\mathrm{SUV}_{\text {mean }}$ error for VOIs averaged over all organs (considering the six VOIs in the lungs as one VOI) is $-12 \pm 10.7 \%$ for PET-MRAC $3 c$ and $-19.0 \pm 7.8 \%$ for PETMRAC4c. Similarly, Fig. 3 shows the relative error between $\mathrm{SUV}_{\text {max }}$ estimated using PET images corrected by MRAC3c and MRAC4c compared to the PET-CTAC for different organs. Table 2 provides a summary of these results. The relative $\mathrm{SUV}_{\max }$ error for VOIs averaged over all body regions is $-9.1 \pm 9.6$ and $-18.4 \pm 8.0 \%$ for PET-MRAC $3 \mathrm{c}$ and PET-MRAC4c, respectively.

Figures 4 and 5 show regression plots and Spearman's correlation analysis between $\mathrm{SUV}_{\text {mean }} / \mathrm{SUV}_{\text {max }}$ for different organs and malignant focal uptake estimated using both MRI-guided attenuation correction techniques and the CTbased attenuation correction technique. The Spearman's correlation coefficient for $\mathrm{SUV}_{\text {mean }}$ metric is equal to 0.97
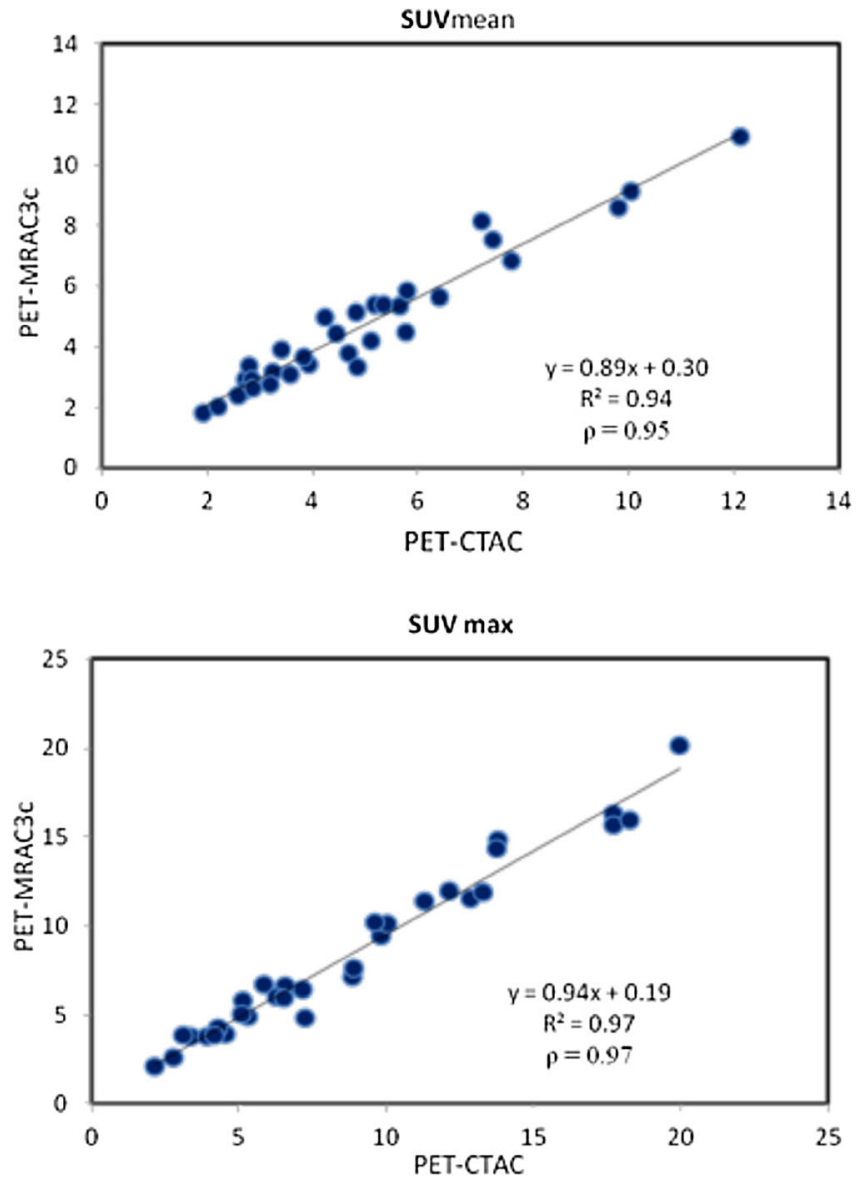

for both PET-MRAC3c and PET-MRAC4c in normal uptake regions and 0.95 and 0.96 for malignant lesions, respectively.

Bland and Altman concordance analysis for $\mathrm{SUV}_{\text {mean }}$ and $\mathrm{SUV}_{\max }$ corresponding to normal physiologic uptake and malignant focal uptake measured on PET-MRAC3c and PET-MRAC4c are shown in Figs. 6 and 7. The limit of agreement and systematic bias (average difference) are presented in the plot.

\section{Discussion}

The 3-class attenuation map discards the air gaps in the inner body and assumes them to be soft tissue (Fig. 8b, top and bottom), whereas the 4-class attenuation map, as implemented in this work, considers the air gaps (Fig. 8c, top and bottom). This resulted in noticeable local SUV differences. For instance, the local SUV in the sinus cavities area is 1.4 on PET-MRAC3c, 0.6 SUV on PET-MRAC4c, and 0.8 SUV on PET-CTAC (Fig. 8 (top)). Similarly, in Fig. 8 (bottom), the local SUV in the air pocket within the abdomen is $1.42,0.32$, and 0.41 for PET-MRAC3c, PETMRAC4c, and PET-CTAC, respectively. Since the approach used to generate the 3-class attenuation map fills the region
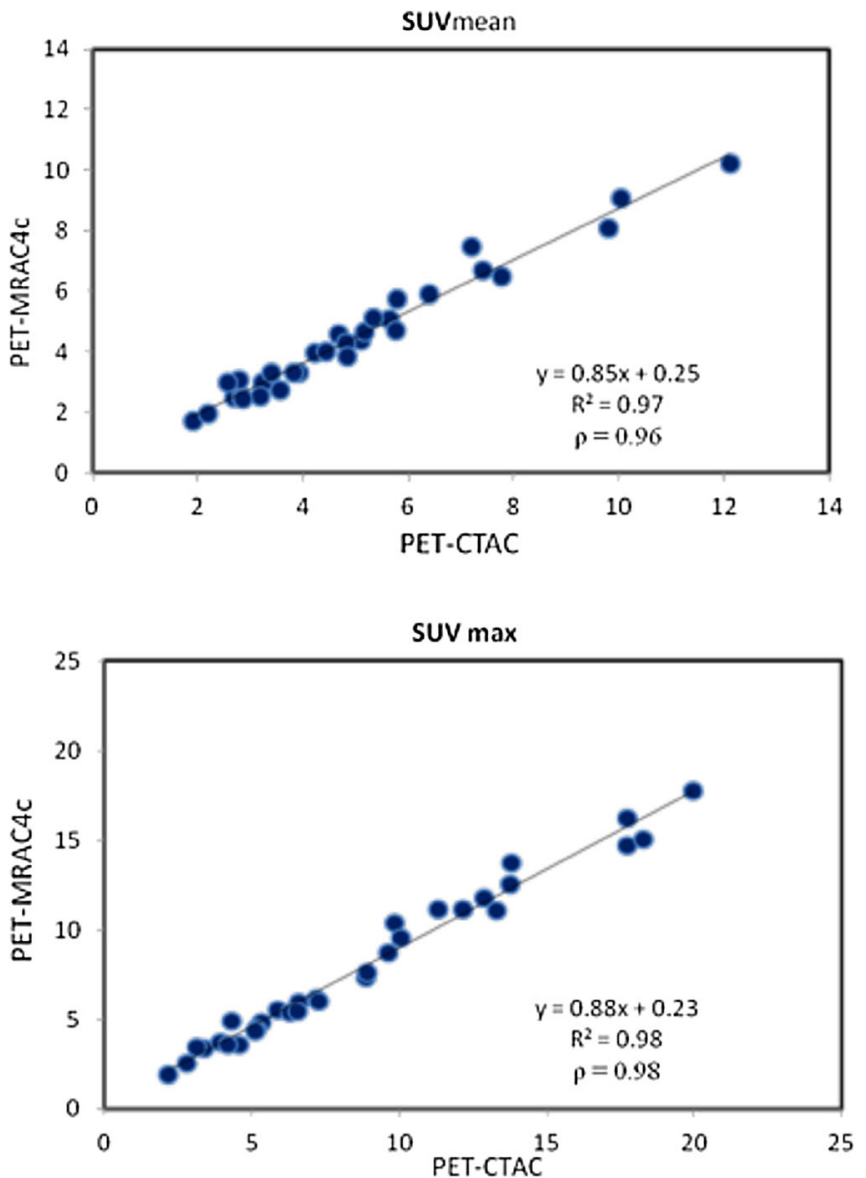

Fig. 5. Regression plots between $\mathrm{SUV}_{\text {mean }} / \mathrm{SUV}_{\max }$ estimated using images corrected for attenuation using 3- (PET-MRAC3c) and 4-class (PET-MRAC4c)-based MR-guided attenuation correction and CT-based attenuation correction (PET-CTAC) for malignant lesions. 

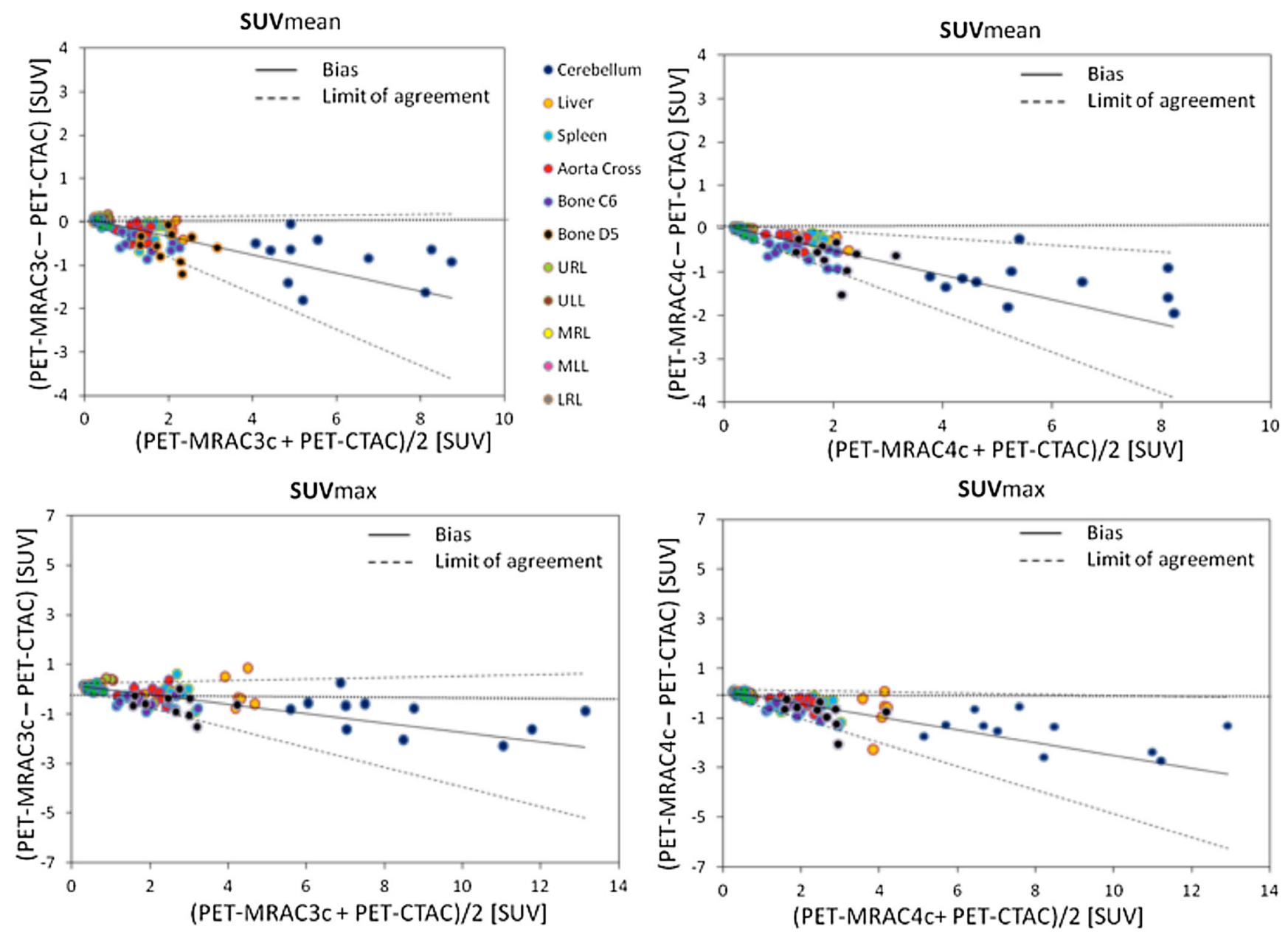

Fig. 6. Bland Altman plots of $S U V_{\text {mean }} / S U V_{\text {max }}$ measurement for various organs between PET-MRAC3c/PET-MRAC4c and PET-CTAC. Bias and limits of agreements are indicated taking into account the relative systematic SUV underestimation. The oblique lines represent results of regression analysis performed on stepwise curves obtained for individual organs.

located inside the external body contour with soft tissue, in contrast to the 4-class attenuation map, it is less sensitive to dental filling and metal implant-related artifacts (Fig. 1d). Although neglecting air cavities in the inner body might result in considerable local SUV bias, filling the inner body void regions with soft tissue leads to more accurate attenuation maps in the presence of metal artifacts on MR images which might affect SUV measurements in PET/MR [41].

Overall, the relative error for $\mathrm{SUV}_{\text {mean }}$ in VOIs defined in normal physiologic uptake, except the lungs (Figs. 3 and 4; Tables 1 and 2), is smaller in the 3-class compared to the 4class attenuation correction technique. The average error for all organs except the lungs is $-17.4 \pm 8.46$ and $-22.0 \pm 6.8 \%$ for PET-MRAC3c and PET-MRAC4c, respectively. The negative bias observed in the liver, cerebellum, and bony regions is consistent with what was reported by Ouyang et al. $(-7.4 \pm 3.4 \%$ for liver and $-15.4 \pm 10.5 \%$ for bone) [22], Schramm et al. ( $-5 \pm 10 \%$ for liver) [21], Kim et al. (-12\% for liver and $-18 \%$ for bone) [42], and Hofmann et al. (-30\% for bone) [14]. Ouyang et al. reported a $5.6 \%$ positive bias in the liver when using a 4-class attenuation map which is inconsistent with our observations. This discrepancy likely stems from the higher soft tissue attenuation coefficient $\left(1.004 \mathrm{~cm}^{-1}\right)$ used in the abovereferenced work. The relative error in bony structures was more severe (Tables 1 and 2), resulting in over $30 \%$ bias owing to neglecting bone in the attenuation correction map [25]. However, the influence of neglecting bone was higher on PET-MRAC4c since bone was mostly replaced with fat tissue owing to partial volume effect in the bone marrow. Our data are in accordance to recently published results which have shown that MRI-guided attenuation mainly underestimates SUVs in areas close to large bony structures $[22,43]$.

The 3-class technique resulted in a mean relative error of $18.6 \pm 15.3 \%$ for all VOIs in the lungs, while the 4-class method yielded an average error of $-0.45 \pm 13.3 \%$ for $\mathrm{SUV}_{\text {mean. }}$ Ouyang et al. [22] observed a positive uptake bias in the lungs for the 3-class attenuation map $(5.8 \pm 14.6 \%)$, while Martinez-Moller et al. [11] reported $-1.8 \%$ bias for the 4-class attenuation map. Since the attenuation 

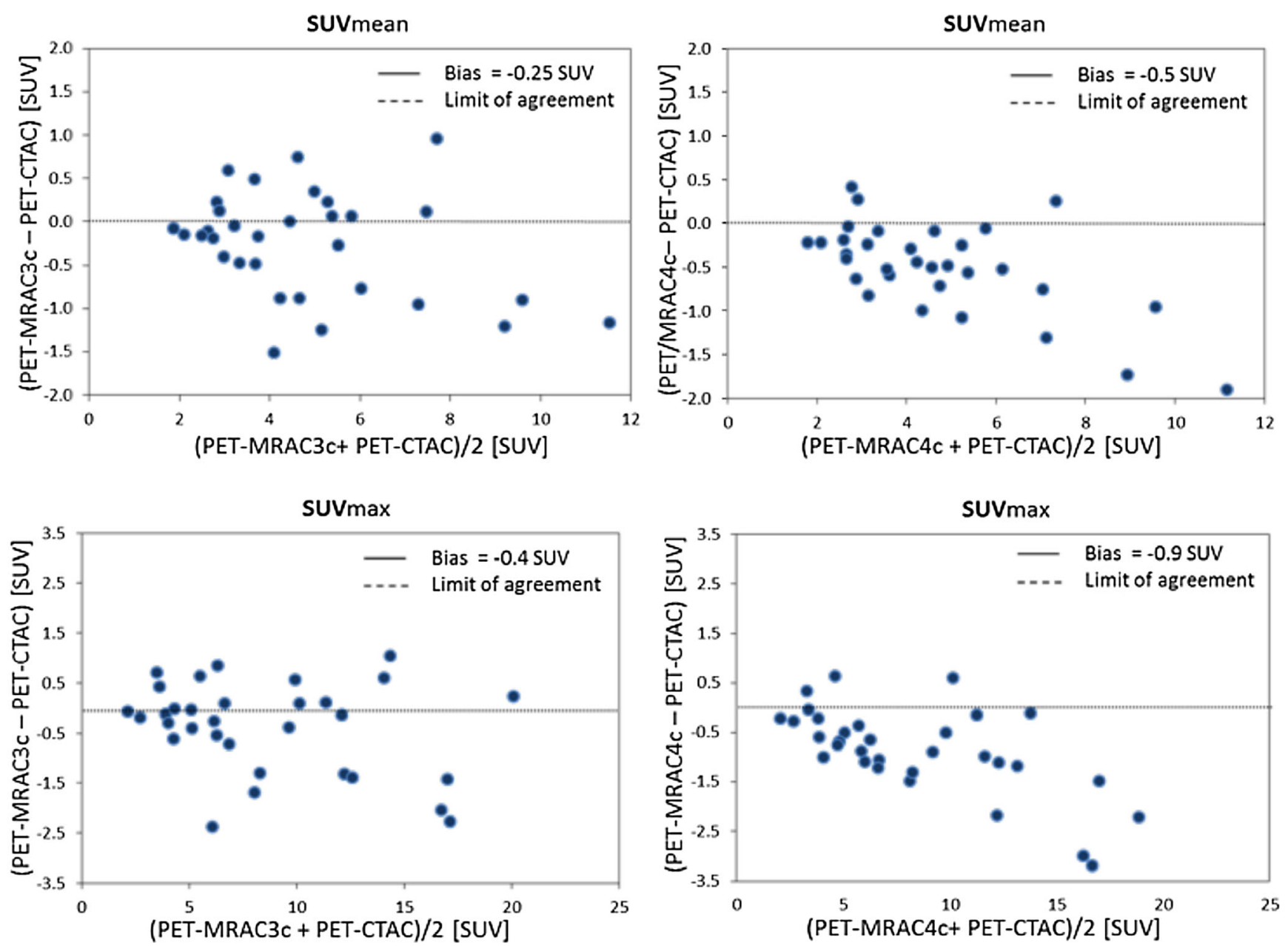

Fig. 7. Bland Altman plots of SUV mean $/ S U V_{\text {max }}$ measurement for malignant lesions between PET-MRAC3c/PET-MRAC4C and PET-CTAC. Bias and limits of agreements are indicated taking into account the relative systematic SUV underestimation.

coefficients assigned to the lung are 0.022 and $0.018 \mathrm{~cm}^{-1}$ in the 3-class and 4-class approaches, respectively, it can be deduced that an attenuation coefficient of $0.018 \mathrm{~cm}^{-1}$ (4class) approximates better attenuation of the lungs at least for our patients. The Shapiro-Wilk test revealed that the null hypothesis of the test was rejected for all but lower left VOIs in the lung for MRAC $3 \mathrm{c}_{\text {mean }}$, whereas it was not rejected for the entire lung VOIs for MRAC4c $\mathrm{c}_{\text {mean }}$. The same trend is observed for $\mathrm{SUV}_{\max }$ (Table 2) where the null hypothesis was not rejected for MRAC4 $\mathrm{c}_{\text {mean }}$ in the lung region. The standard deviation for both techniques in the lung region was approximately similar which indicates comparable correlation strength with mean values. The density of the lungs varies between patients due to respiratory motion, smoking habits, or age [44]. The 3-class and 4-class attenuation correction techniques resulted in -3.9 and $-9.2 \%$ relative errors for malignant lesions (Fig. 2), respectively. Since most patients included in this study protocol had lesions located in the head and neck region, the characteristics of the bias for lesions follow a similar trend to VOIs located outside the lungs where neglecting bony structures caused underestimation of tracer uptake. None of the patients had lesions in the lungs. Since a positive bias was obtained for VOIs located in the lungs when using PETMRAC3c whereas a small negative bias was obtained for PET-MRAC4c, the bias for lesions located in the lungs might be different between these two attenuation correction techniques. The Shapiro-Wilk test (Tables 1 and 2) demonstrates that the null hypothesis was rejected for lesions and normal physiologic regions (except the lungs), whereas the $P$ values are not statistically significant for the liver and spleen when using MRAC $3 \mathrm{c}_{\max }$.

The relative errors for VOIs defined in the lungs were considerably high (Tables 1 and 2); however, the absolute differences indicate insignificant errors for both PETMRAC3c and PET-MRAC4c (Supplemental Figs. S2, S3, and S4). The average absolute SUV differences for all VOIs in the lung region are 0.05 and 0.02 for $\mathrm{SUV}_{\text {mean }}$, while they are -0.08 and -0.03 for $\mathrm{SUV}_{\max }$ for PET-MRAC3c and PET-MRAC4c, respectively. This is due to the low tracer uptake in the lungs and the noise associated with image reconstruction in low-count regions [14]. The maximum absolute difference for VOIs defined in regions of normal physiologic uptake occurred in the cerebellum for both PET- 

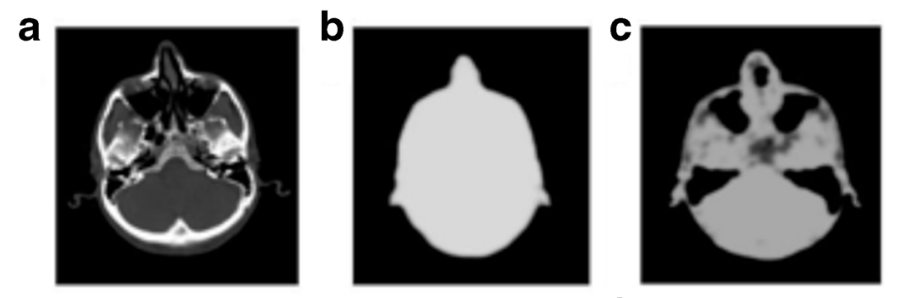

d
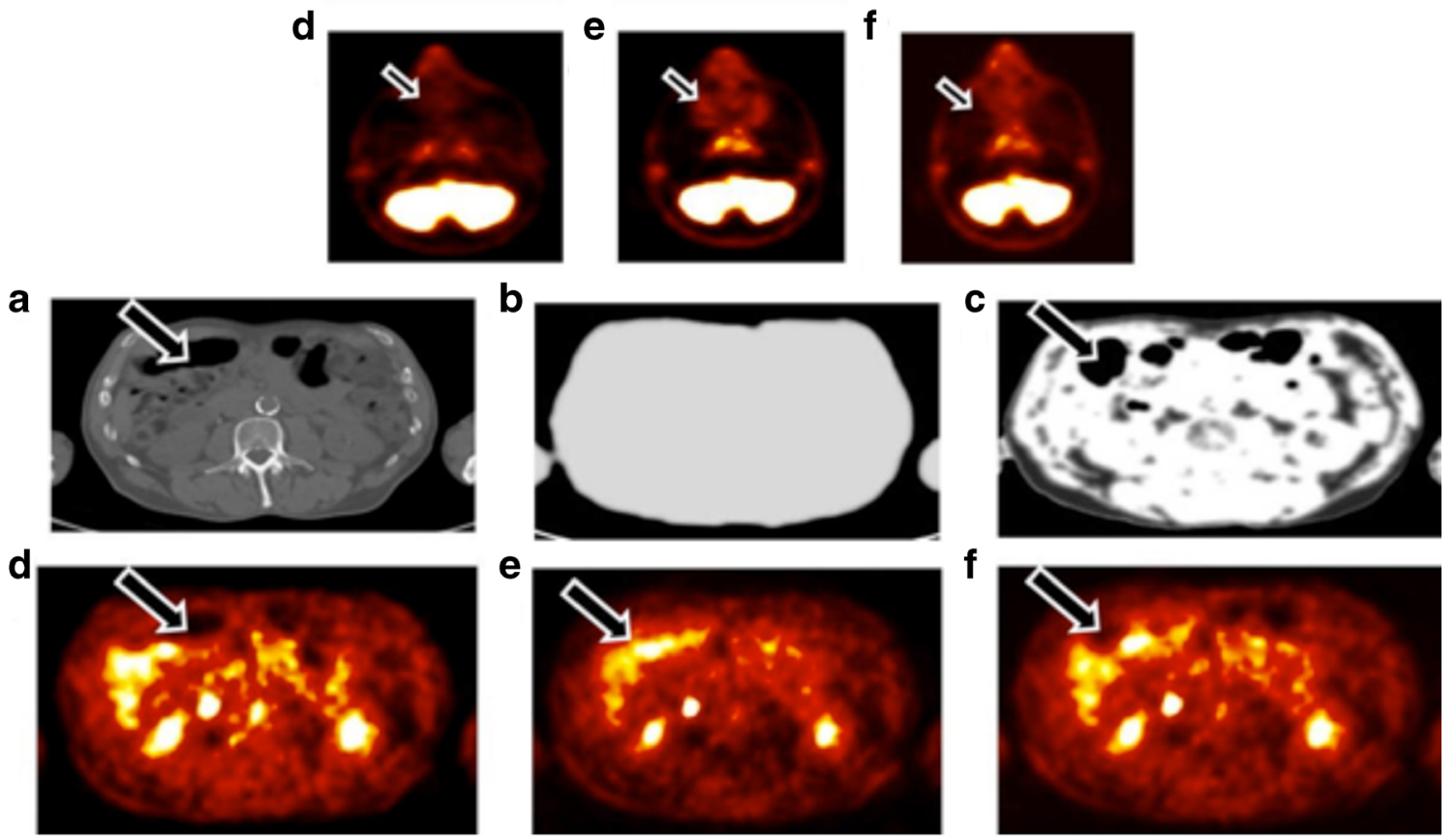

Fig. 8. Representative slice showing the impact of neglecting sinus air cavities (top) and air pockets in the abdomen (bottom) in the 3-class attenuation map. a CT, b MRAC3c attenuation map, c MRAC4c attenuation map, d PET-CTAC, e PET-MRAC3c, and $\mathbf{f}$ PET-MRAC4c. SUVs measured in sinus cavities and air pockets are 0.80 and $0.41 \mathrm{SUV}_{\text {mean }}$ on PET-CTAC, 1.40 and 1.42 SUV $_{\text {mean }}$ on PET-MRAC3c, and 0.60 and 0.32 SUV $_{\text {mean }}$ on PET-MRAC4c, respectively.

MRAC3c and PET-MRAC4c. A plausible explanation of this negative bias might be that the thickness and density of ignored bony structures (skull) around the cerebellum is significantly higher than in other organs. The same observations were made by Bezrukov et al. [9] and Ouyang et al. [22] reporting a negative SUV bias of up to $-12 \%$ near bony regions. In contrast, Schramm et al. [21] reported $12 \%$ SUV bias in the cerebellum. The average absolute errors for lesions are 0.25 and 0.5 (maximum -2.4) for $\mathrm{SUV}_{\text {mean }}$, while they are 0.4 and 0.86 (maximum -3.2) for $\mathrm{SUV}_{\max }$ for PET-MRAC3c and PET-MRAC4c, respectively. The lesion located near bony structures resulted in a higher absolute error particularly when using the 4-class attenuation map owing to the misclassification of bone marrow as fat.

The high Spearman's correlation coefficient ( $\rho=0.97$ for both techniques) indicates a statistically significant relationship between SUVs measured on PET-MRAC3c/PETMRAC4c and PET-CTAC (Fig. 4). However, it should be noted that a significant non-zero correlation coefficient does not imply that these attenuation correction approaches are concordant, since the probability that they deviate from each other remains even in case of strong correlation. Consequently, Bland and Altman analysis was performed to depict by how much the SUVs measured on PET images corrected for attenuation using both approaches are likely to deviate from the SUVs measured on PET-CTAC. Bland and Altman plots indicate that the systematic bias was higher on PETMRAC3c than PET-MRAC4c for normal physiologic uptake (except the lungs), while $\mathrm{SUV}_{\max }$ had lower bias than $\mathrm{SUV}_{\text {mean. }}$ The systematic bias was higher on PETMRAC4c than PET-MRAC3c for malignant lesions. It worth mentioning that, in accordance to data published by previous investigators [5], the change in SUV measurements depends on their absolute values. That is, the higher the SUVs, the higher the absolute difference between SUVs measured on PET-MRAC and PET-CTAC images.

\section{Conclusion}

In this work, we evaluate the quantitative accuracy of the 3class and 4-class attenuation correction techniques used on hybrid PET/MRI systems. Overall, the quantitative analysis revealed significant underestimation of SUVs in organs presenting with normal physiologic uptake (except the lungs) and malignant lesions by both PET-MRAC3c and PET-MRAC4c as compared to PET-CTAC. The 3-class 
technique resulted in overestimation of tracer uptake in the lung, whereas the 4-class approach produced insignificant negative bias. This trend is explained by the different attenuation coefficients for lung tissue used by both attenuation correction techniques. The largest differences were observed in bony structures or near massive bones for both approaches. Despite the bias induced by both attenuation correction approaches, there is good correlation between SUVs measured on PET-MRAC images and PETCTAC. Overall, it was observed that the 3-class attenuation map generates a lower systematic negative bias of tracer uptake; however, the attenuation coefficient assigned to the lung should be adapted. Regardless of the attenuation correction technique used, it is strongly recommended to check the attenuation map as part of the reading procedure for the presence of artifact, which might in some cases induce clinically relevant SUV changes, including metal artifacts, lung and body contour segmentation errors, and miss-alignment issues.

Acknowledgments. This work was supported by the Swiss National Science Foundation under grants SNSF 31003A-135576, SNFN 31003A-149957, and SNSF 320030 135728/1.

Conflict of Interest. The authors declare that they have no conflict of interest.

\section{References}

1. Judenhofer MS, Wehrl HF, Newport DF et al (2008) Simultaneous PET-MRI: a new approach for functional and morphological imaging. Nat Med 14:459-465

2. Zukotynski KA, Fahey FH, Kocak M et al (2011) Evaluation of 18FFDG PET and MRI associations in pediatric diffuse intrinsic brain stem glioma: a report from the pediatric brain tumor consortium. J Nucl Med 52:188-195

3. Hirsch FW, Sattler B, Sorge I et al (2013) PET/MR in children. Initial clinical experience in paediatric oncology using an integrated PET/MR scanner. Pediatr Radiol 43:860-875

4. Zaidi H, Del Guerra A (2011) An outlook on future design of hybrid PET/MRI systems. Med Phys 38:5667-5689

5. Varoquaux A, Rager O, Poncet A et al (2014) Detection and quantification of focal uptake in head and neck tumours: (18)F-FDG PET/MR versus PET/CT. Eur J Nucl Med Mol Imaging 41:462-475

6. Wiesmuller M, Quick HH, Navalpakkam B et al (2013) Comparison of lesion detection and quantitation of tracer uptake between PET from a simultaneously acquiring whole-body PET/MR hybrid scanner and PET from PET/CT. Eur J Nucl Med Mol Imaging 40:12-21

7. Becker M, Zaidi H (2014) Imaging in head and neck squamous cell carcinoma: the potential role of PET/MRI. Br J Radiol 87:20130677

8. Zaidi H (2007) Is MRI-guided attenuation correction a viable option for dual-modality PET/MR imaging? Radiology 244:639-642

9. Bezrukov I, Mantlik F, Schmidt H et al (2013) MR-based PET attenuation correction for PET/MR imaging. Semin Nucl Med 43:45-59

10. Zaidi H, Montandon M-L, Slosman DO (2003) Magnetic resonance imaging-guided attenuation and scatter corrections in three-dimensional brain positron emission tomography. Med Phys 30:937-948

11. Martinez-Moller A, Souvatzoglou M, Delso G et al (2009) Tissue classification as a potential approach for attenuation correction in whole-body PET/MRI: evaluation with PET/CT data. J Nucl Med 50:520-526

12. Schulz V, Torres-Espallardo I, Renisch S et al (2011) Automatic, threesegment, MR-based attenuation correction for whole-body PET/MR data. Eur J Nucl Med Mol Imaging 38:138-152

13. Montandon M-L, Zaidi H (2005) Atlas-guided non-uniform attenuation correction in cerebral 3D PET imaging. Neuroimage 25:278-286
14. Hofmann M, Bezrukov I, Mantlik F et al (2011) MRI-based attenuation correction for whole-body PET/MRI: quantitative evaluation of segmentation- and atlas-based methods. J Nucl Med 52:1392-1399

15. Catana C, van der Kouwe A, Benner T et al (2010) Toward implementing an MRI-based PET attenuation-correction method for neurologic studies on the MR-PET brain prototype. J Nucl Med 51:1431-1438

16. Keereman V, Fierens Y, Broux T et al (2010) MRI-based attenuation correction for PET/MRI using ultrashort echo time sequences. J Nucl Med 51:812-818

17. Berker Y, Franke J, Salomon A et al (2012) MRI-based attenuation correction for hybrid PET/MRI systems: A 4-class tissue segmentation technique using a combined ultrashort-echo-time/Dixon MRI sequence. J Nucl Med 53:796-804

18. Salomon A, Goedicke A, Schweizer B et al (2011) Simultaneous reconstruction of activity and attenuation for PET/MR. IEEE Trans Med Imaging 30:804-813

19. Defrise M, Rezaei A, Nuyts J (2012) Time-of-flight PET data determine the attenuation sinogram up to a constant. Phys Med Biol 57:885-899

20. Rezaei A, Defrise M, Bal G et al (2012) Simultaneous reconstruction of activity and attenuation in time-of-flight PET. IEEE Trans Med Imaging 31(12):2224-2233

21. Schramm G, Langner J, Hofheinz F et al (2013) Quantitative accuracy of attenuation correction in the Philips Ingenuity TF whole-body PET/ MR system: a direct comparison with transmission-based attenuation correction. Magn Reson Mat Phys Biol Med 26:115-126

22. Ouyang J, Chun SY, Petibon Y et al (2013) Bias atlases for segmentation-based PET attenuation correction using PET-CT and MR. IEEE Trans Nucl Sci 60:3373-3382

23. Samarin A, Burger C, Wollenweber SD et al (2012) PET/MR imaging of bone lesions-implications for PET quantification from imperfect attenuation correction. Eur J Nucl Med Mol Imaging 39:11541160

24. Keereman V, Holen RV, Mollet P, Vandenberghe S (2011) The effect of errors in segmented attenuation maps on PET quantification. Med Phys 38:6010-6019

25. Akbarzadeh A, Ay MR, Ahmadian A et al (2013) MRI-guided attenuation correction in whole-body PET/MR: assessment of the effect of bone attenuation. Ann Nucl Med 27:152-162

26. Burgos N, Cardoso MJ, Modat M et al (2013) Attenuation correction synthesis for hybrid PET-MR scanners. Med Image Comput Comput Assist Interv 16:147-154

27. Zaidi H, Ojha N, Morich M et al (2011) Design and performance evaluation of a whole-body Ingenuity TF PET-MRI system. Phys Med Biol 56:3091-3106

28. Dixon WT (1984) Simple proton spectroscopic imaging. Radiology 153:189-194

29. Delso G, Furst S, Jakoby B et al (2011) Performance measurements of the Siemens mMR integrated whole-body PET/MR scanner. J Nucl Med 52:1914-1922

30. Eiber M, Martinez-Moller A, Souvatzoglou M et al (2011) Value of a Dixon based MR-PET attenuation correction sequence for the localization and evaluation of PET positive lesions. J Nucl Med 52:105

31. Klein S, Staring M, Murphy K et al (2010) Elastix: a toolbox for intensity-based medical image registration. IEEE Trans Med Imaging 29:196-205

32. Akbarzadeh A, Gutierrez D, Baskin A et al (2013) Evaluation of wholebody MR to CT deformable image registration. J Appl Clin Med Phys $14: 238-253$

33. Hu Z, Ojha N, Renisch S, et al. (2009) MR-based attenuation correction for a whole-body sequential PET/MR system. IEEE Nuclear Science Symposium \& Medical Imaging Conference. 25-31 October 2009, Orlando (FL), USA: IEEE; 2009, p 3508-12

34. Kass M, Witkin A, Terzopoulos D (1988) Snakes: active contour models. Int J Comput Vision 1:321-331

35. Yushkevich PA, Piven J, Hazlett HC et al (2006) User-guided 3D active contour segmentation of anatomical structures: significantly improved efficiency and reliability. Neuroimage 31:1116-1128

36. Otsu N (1979) A threshold selection method from gray-level histograms. IEEE Trans Sys Man Cyber 9:62-66

37. Huang SC (2000) Anatomy of SUV. Standardized uptake value. Nucl Med Biol 27:643-646

38. Bland JM, Altman DG (1986) Statistical methods for assessing agreement between two methods of clinical measurement. Lancet 1:307-310 
39. Gönen M, Panageas KS, Larson SM (2001) Statistical issues in analysis of diagnostic imaging experiments with multiple observations per patient1. Radiology 221:763-767

40. Galbraith S, Daniel JA, Vissel B (2010) A study of clustered data and approaches to its analysis. J Neurosci 30:1060110608

41. Ladefoged CN, Andersen FL, Keller SH et al (2013) PET/MR imaging of the pelvis in the presence of endoprostheses: reducing image artifacts and increasing accuracy through inpainting. Eur J Nucl Med Mol Imaging 40:594-601
42. Kim JH, Lee JS, Song IC, Lee DS (2012) Comparison of segmentationbased attenuation correction methods for PET/MRI: evaluation of bone and liver standardized uptake value with oncologic PET/CT data. J Nucl Med 53:1878-1882

43. Bini J, Izquierdo-Garcia D, Mateo J et al (2013) Preclinical evaluation of MR attenuation correction versus CT attenuation correction on a sequential whole-body MR/PET scanner. Invest Radiol 48:313-322

44. Soejima K, Yamaguchi K, Kohda E et al (2000) Longitudinal follow-up study of smoking-induced lung density changes by high-resolution computed tomography. Am J Respir Crit Care Med 161:1264-1273 